\title{
Preface of the special issue: the 6th International Conference on Optimization and Control with Applications (6th OCA)
}

\author{
Kok Lay Teo ${ }^{1} \cdot$ Adil Bagirov $^{2} \cdot$ Wuyi Yue ${ }^{3}$ \\ Received: 10 July 2018 / Accepted: 13 July 2018 / Published online: 19 July 2018 \\ (c) Springer-Verlag GmbH Germany, part of Springer Nature 2018
}

This volume of the Optimization Letters is devoted to papers presented at the 6th International Conference on Optimization and Control with Applications (6th OCA), which was held on December 11-14, 2015, at International Academic Exchange Center of Changsha University of Science and Technology, Changsha, China.

The conference attracted more than 300 registered participants. They exchanged experiences in solving real-world problems, discussed recent achievements in optimization and control theory, reported on developments and implementations of efficient solution procedures for problems of optimization and control. The 6th OCA provided an excellent forum for researchers and practitioners to promote their recent advances in optimization and control to the wider scientific community, to identify new research challenges as well as promising research developments in theory, methods and applications.

For this special issue, participants of the 6th OCA were invited to submit papers on optimization, control and related topics. The papers included recent theoretical and applied contributions in various fields of optimization as well as control theory. Based on rigorous reviewing processes, 19 papers were accepted for publication and included in this special issue. Their short descriptions follow.

$凶$ Kok Lay Teo

k.1.teo@curtin.edu.au

Adil Bagirov

a.bagirov@federation.edu.au

Wuyi Yue

yue@konan-u.ac.jp

1 School of Electrical Engineering, Computing and Mathematical Sciences, Faculty of Science and Engineering, Curtin University of Technology, GPO Box U1987, Perth, WA 6845, Australia

2 School of Science, Information Technology \& Engineering, Federation University Australia, University Drive, Mount Helen, P.O. Box 663, Ballarat, VIC 3353, Australia

3 Department of Information Science \& Systems Engineering, Faculty of Science and Engineering, Konan University, 8-9-1 Okamoto, Higashinada-Ku, Kobe 658-8501, Japan 
Wang and Zhang study an interior penalty method for a finite-dimensional large-scale linear complementarity problem arising often from the discretization of stochastic optimization problems in financial engineering. Wu et al. develop a distributed algorithm utilizing the subgradient information with stochastic errors to solve a distributed optimization problem encountered in a time varying multi-agent network. Zhang et al. study sliding mode control problems for discrete-time descriptor Markovian jump systems which contain two mutually independent homogeneous Markov chains.

Liu et al. propose a bi-objective dynamic optimization model using a nonlinear timedelay system to optimize the 1,3-propanediol (1,3-PD) production in a microbial batch process. Sun et al. report Farkas-type results for a fractional programming problem using the properties of dualizing parametrization functions, Lagrangian functions and the epigraph of the conjugate functions. Li et al. consider zero-forcing beamforming under per-antenna power constraints for rural applications and propose a reduced complexity zero-forcing beam-former design.

Li and Wang establish various properties and characterizations of the set of all robust optimal solutions of the convex programming problems with data uncertainty in both the objective function and the constraints. Wang et al. establish sufficient conditions for the zero duality gap property between a optimal control problem, where the state of the system is defined by a mixed quasi-variational inequality, and its nonlinear dual problem using nonlinear Lagrangian methods. Peng et al. consider semi-infinite vector optimization problems under functional perturbations of both objective functions and constraint sets, establish the Berge-lower semicontinuity and Painleve-Kuratowski convergence of the constraint set mapping and use them to obtain sufficient conditions of Painleve-Kuratowski stability for approximate efficient and approximate weakly efficient solution mappings.

Sun et al. study distributionally robust two-stage stochastic linear optimization problems with higher-order moment constraints in their ambiguity sets and propose to solve the dual form of the problem by a semi-infinite programming approach. Zhou et al. introduce a notion of the semi-E cone convex set-valued map in locally convex spaces, investigate the existence conditions of the weakly efficient element of the set-valued optimization problem and establish that the local weakly efficient element of the set-valued optimization problem is the weakly efficient element. Song et al. consider non-zero-sum regular-singular stochastic differential games, study the control strategy of each player, which consists of two components: regular control and singular control, and apply the Malliavin calculus approach to establish a necessary maximum principle for the games.

Ding et al. present results on a tractable convex programming approximation for distributionally robust individual chance constrained problem under interval sets of mean and covariance information. They demonstrate that the worst-case CVaR approximation problem is an exact form of the distributionally robust individual chance constrained problem. Tawhid and Rahmati investigate uniqueness, feasibility, and strict feasibility of the solution of a complementarity problem induced by a (compact) 
set of hypermatrices. Yu et al. investigate the effect of yield uncertainty in price-setting newsvendor models involving an additive-multiplicative demand.

Zhao and Yue analyse a two-dimensional discrete-time Markov chain that represents the cognitive radio networks with buffer settings and obtain formulas for several system performance measures. Liu et al. present a novel adaptive spectrum reservation strategy in Cognitive Radio Networks and demonstrate its performance. Li et al. investigate saddle points and gap functions for weak generalized Ky Fan inequalities. Long et al. establish sufficient and necessary global optimality conditions for fixed charge quadratic programming problem using the concept of abstract convexity.

Acknowledgements We, as guest editors, would like to thank all authors who showed interest in this special issue and all the reviewers who accepted the invitation to provide their expert and constructive comments. Thanks to the Editors-in-Chief of Optimization Letters journal, P. Krokhmal and O.A. Prokopyev, for their interest and continuous support. We hope that the reader will find the reading of the contributions of this special issue enjoyable and inspiring. 